\title{
Board 62: Work In Progress for Developing Project-Based Experiential Learn- ing of Engineering Electromagnetics
}

\section{Mr. Adriyel Nieves, The Pennsylvania State University}

Adriyel Nieves is currently pursuing a Masters degree in electrical engineering at The Pennsylvania State University. He worked as a professional engineer for three years after receiving his bachelors degree in electrical engineering from Penn State in 2015. His interests include radar systems, Electromagnetic theory, meta-materials, antenna design, embedded systems, and improving engineering education.

\section{Dr. Julio Urbina, Pennsylvania State University, University Park}

JULIO V. URBINA, Ph.D is an Associate Professor in the School of Electrical Engineering and Computer Science at Penn State. His educational research interests include effective teaching techniques for enhancing engineering education, global engineering and international perspectives, thinking and working in multi-, inter-, and transdisciplinary ways, cyberlearning and cyber-environments, service and experiential learning, teaming and collaborative learning.

\section{Prof. Tim Kane, Pennsylvania State University, University Park}

TMOTHY J. KANE, Ph.D is Professor of Electrical Engineering and Meteorology at Penn State who teaches the undergraduate engineering electromagnetics course, EE 330. His educational research interests include developing course materials for enhancing engineering education, collaborative learning, and increasing student involvement.

\section{Prof. Shengxi Huang, The Pennsylvania State University}

Mr. Diego Penaloza , Pennsylvania State University, University Park

Received the B.S. degree in electronics engineering from the Universidad Nacional de Ingenieria, Lima, Peru, in 2016. From 2017 to 2018, worked as a research assistant at the National Research and Training Institute of Telecommunications (INICTEL-UNI), Lima. He is currently pursuing the Ph.D. degree in the department of Electrical Engineering at Penn State University, University Park, PA, USA. He is currently a research assistant in the Applied Signal Processing and Instrumentation Research Laboratory (ASPIRL) in the same department. His research interests include applied electromagnetics, antennas, cognitive radars, software- defined radio and remote sensing. 


\section{Work in Progress for Developing Project-Based Experiential Learning of Engineering Electromagnetics}

\section{Introduction}

Electromagnetics (EM) is traditionally taught as a core course within the electrical engineering curriculum. The theory and application of EM has enabled engineers and scientists to develop applications such as Radio Frequency (RF) circuits, telecommunication systems, and radar systems. Similarly, scientists have been able to characterize various phenomena in nature with EM theory such as the behavior and application of plasmonic material. Over the last decade, there has been a decrease in the interest of electrical engineering students in taking more elective EM courses within our curriculum. Industry has witnessed these effects and has collaborated with various universities to define a standard pipeline to train students at the university level to fill gaps within the workforce [1].

As a solution, this paper is the work in progress for a holistic hands-on activity leading to the completion of a final project for the course, which is the implementation of a low-power continuous wave radar. This instrument has many components that can be linked to the fundamentals of electromagnetic theory. In addition, to build a functional radar sensor, other fundamental electrical engineering topics such as electronic circuits, power systems, signal processing, etc. are needed. Previous research in designing laboratory experiments has shown that the "final project concept" is a very successful method to engage students to learn engineering concepts $[2,3,4]$. To prepare the students for the final project of our EM course, 7 laboratory experiments have been developed to follow the classroom lectures so students can reinforce content from each lecture through hands-on experience.

\section{Designing the Laboratory}

The following steps were taken to develop the laboratory experiments. First, prototype tools and experiments were developed as proofs of concept. Second, an analysis of course content was compared with the goals of the laboratory experiments to ensure that the course content matched with the flow of the projects. Third, the laboratory write-ups were developed with an emphasis on making the content engaging and rewarding for the student to learn a meaningful application. Fourth, the lab-write ups and experiments were finalized. Partial testing through the current semester and results will be finalized at the end of the semester. 


\section{Step 1: System Prototype}

A radar system has many components spanning multiple fields within the electrical engineering undergraduate curriculum. A kit was used to demonstrate the feasibility of building a radar system from simple components [5]. The kit is comprised of circuit components to build a waveform generator and a 2-stage video amplifier, mini-circuit components to build the RF circuit, two cylindrical waveguides fashioned from coffee cans, and required connectors to piece the system together. The kit is powered by $8 \mathrm{AA}$ batteries which provide $12 \mathrm{~V}$ and $5 \mathrm{~V}$ to the circuitry. All the components can be viewed as a "black-box" for the purpose of abstraction for concepts that may be introduced later in the course or that may be out of the scope at the junior level EM course.

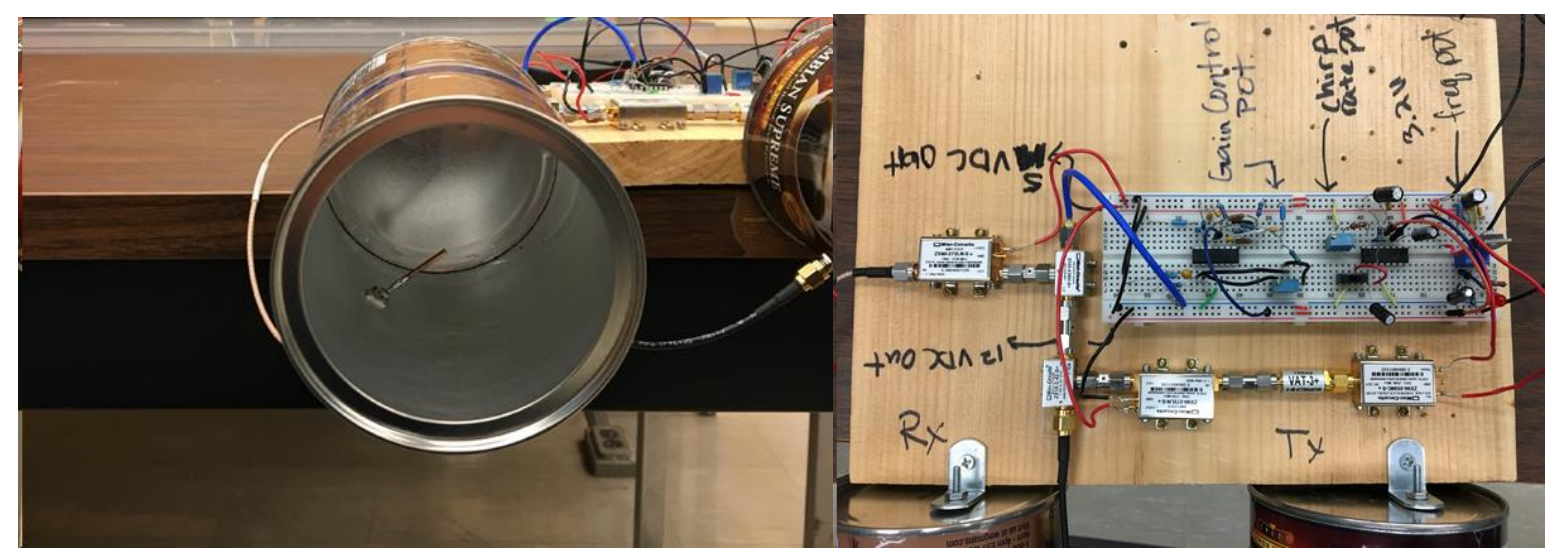

Figure 1. (Left) Coffee cans used as an antenna, (right) Physical layout of the radar system.

\section{Step 2: Course Analysis Content}

It is vital that laboratory experiments correspond with course lecture topics enabling students to make a concrete mental connection. Order and content are vital in the understanding of electromagnetic theory. Within the collaborators, we discussed the best method based on experience of teaching the course on how to coordinate the course content and lab experiments.

For a typical undergraduate just introduced to Maxwell's equations, the suggested progression is to develop an understanding of static fields, then progress into dynamic fields [6]. Many textbooks have adopted this approach which is advantageous as the student gets an understanding of the interaction between the physics and mathematics. The downfall to this approach is the student can lose 
insight if too much time is focused on static fields [6]. Since students at our university already have a background in electrostatics and magnetostatics, these topics are compressed in the curriculum to emphasize new topics in more detail.

The following table is the suggested structure of the course based on the textbook utilized within the EM course and the flow of the project goal. The length of time determined to spend on each topic is dependent on the importance of the topic to electrical engineering, if the topic was covered in a prerequisite course, and the difficulty of the topic. There is a paired laboratory experiment suggested to correspond with the topics covered in one week (two lectures).

Table 1. 15-Week course layout and associated student lab experiment. (try to keep table on same page)

\begin{tabular}{|c|c|c|}
\hline Week & Topics & Recitation/Computing/Lab/Comments \\
\hline 1 & Introduction & Lab \#1: MATLAB Introduction \\
\hline 2 & Transmission Lines & $\begin{array}{l}\text { Recitation: Complex Numbers and } \\
\text { Phasors }\end{array}$ \\
\hline 3 & Transmission Lines (Cont.) & $\begin{array}{l}\text { Lab \#2: EM Tools and Transmission } \\
\text { Line effects at RF }\end{array}$ \\
\hline 4 & Vector Analysis Review & $\begin{array}{l}\text { Lab \#3: RF Lab Components and } \\
\text { Theory }\end{array}$ \\
\hline 5 & Electrostatics/Magnetostatics & Recitation Review \\
\hline 6 & $\begin{array}{l}\text { Maxwell's Equations (time- } \\
\text { varying) }\end{array}$ & Lab \#4: Part 1: Anechoic Chamber \\
\hline 7 & Maxwell's Equations (Cont.) & Recitation: Maxwell's Equations \\
\hline 8 & Radiation and Antennas & Lab \#4: Part 2: Antenna Radiation \\
\hline 9 & $\begin{array}{l}\text { Plane Wave Propagation and } \\
\text { Polarization }\end{array}$ & $\begin{array}{l}\text { Lab \#5: Plane Waves Propagation and } \\
\text { Polarization }\end{array}$ \\
\hline 10 & $\begin{array}{l}\text { Reflection \& Transmission... } \\
\text { \& Waveguides }\end{array}$ & $\begin{array}{l}\text { Lab \#6: Reflection and Transmission of } \\
\text { EM waves }\end{array}$ \\
\hline 11 & EM Applications (Radars) & Lab \#7: Waveguide Lab \\
\hline 12 & $\begin{array}{l}\text { EM Applications (Radars } \\
\text { cont.) }\end{array}$ & $\begin{array}{l}\text { Final Project: Building a Radar } \\
\text { (Integration) }\end{array}$ \\
\hline 13 & $\begin{array}{l}\text { EM Applications (Cell } \\
\text { Phones) }\end{array}$ & $\begin{array}{l}\text { Final Project: Building a Radar } \\
\text { (Analysis of Components) }\end{array}$ \\
\hline 14 & $\begin{array}{l}\text { EM Applications (Cell } \\
\text { Phones Cont.) }\end{array}$ & $\begin{array}{l}\text { Final Project: Building a Radar } \\
\text { (Measurements) }\end{array}$ \\
\hline 15 & EM Applications & $\begin{array}{l}\text { Final Project: Building a Radar } \\
\text { (Analysis and Interpretation) }\end{array}$ \\
\hline
\end{tabular}




\section{Step 3: Lab Write-up Development}

The lab handout has four main components. The first is the motivation and application of the topic to lecture, the second is the pre-lab work (the student must understand necessary theory prior to lab), the third is the actual lab content with 'exploratory' hands-on experiments and open-ended questions that test the knowledge, and the fourth is a reflection and corresponding write-up to the experiment.

A radar is selected as the project because it represents a model for many electromagnetic topics taught within a typical course. Additionally, there are many enthusiast and open source components radar endeavors available [7,8]. Our radar has components based on complex theory. Each lab introduces a concept that is used to understand a portion of a radar. Figure 2 demonstrates how each portion of the radar corresponds with the lecture topics and relationship to other topics covered in other core courses in our curriculum. Any portion of the radar that does not fit within the EM course is introduced to the students as a black box where the interaction of the inputs and outputs pertaining to the radar are studied.

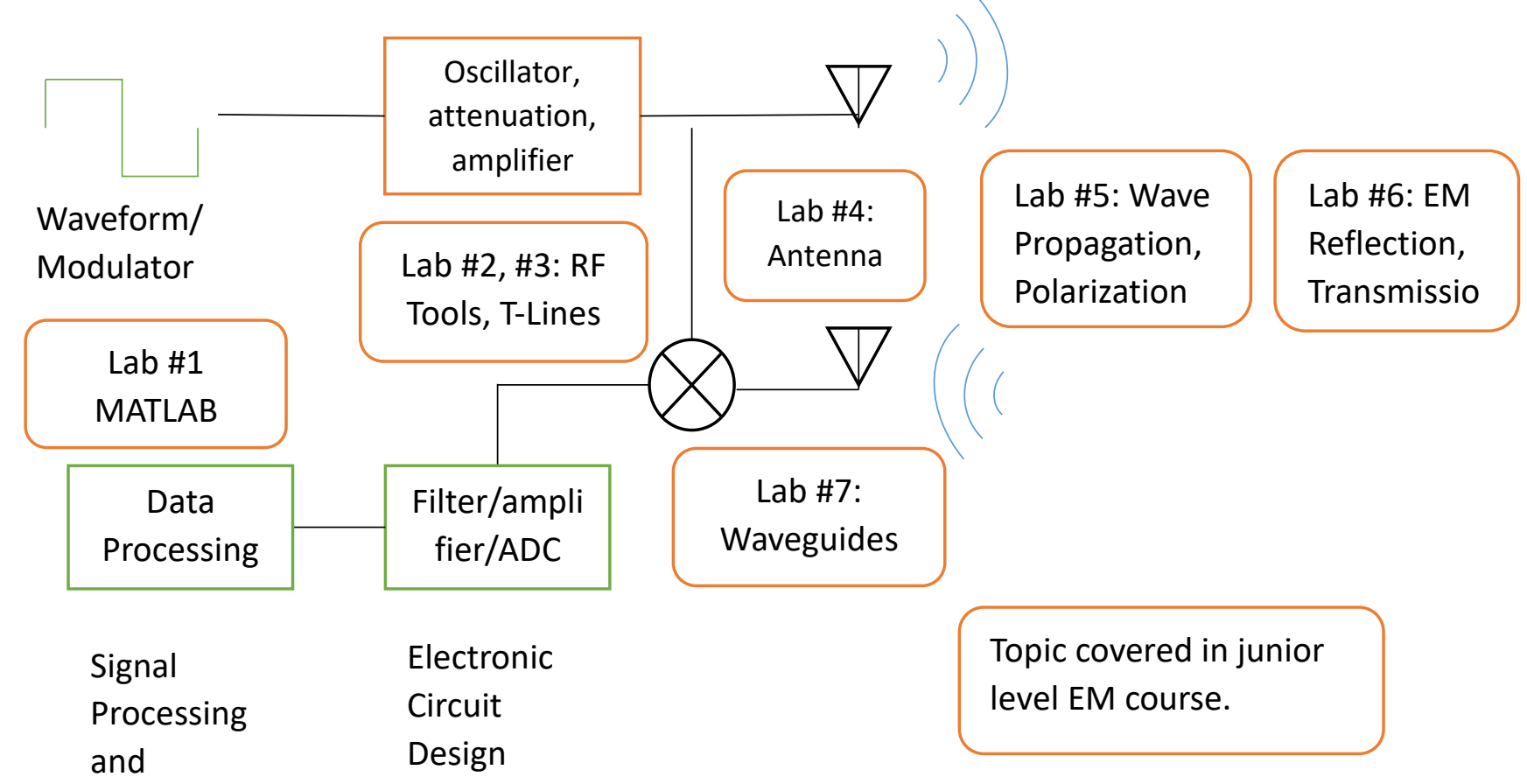

Algorithms

Figure 2. Conceptual Block Diagram of the Radar System.

A waveform generator circuit is built to feed into the voltage-controlled oscillator. Based on the frequency of the waveform, the oscillator generates an 
electromagnetic signal which is fed into an RF chain. The signal is sent to a transmitting monopole antenna surrounded by a cylindrical waveguide. The monopole is designed to operate at a frequency of $2.45 \mathrm{GHz}$ and excites the waveguide at the fundamental mode. The wave propagates into free space and is reflected and/or transmitted based on the target. The target reflection radiates back to the receiver antenna which is detected by a different monopole using a cylindrical waveguide. The signal is mixed with the transmitted wave and fed to a low pass filter to get the difference. Additional circuitry is used to filter the wave to be transmitted into the soundcard of a computer. The data can now be accessed using an open source audio program to get the digitized waveform. This is then processed using MATLAB algorithms to create target range plots, target velocity, and SAR imaging [5]. A short description of each lab is given in appendix A.

\section{Laboratory Description}

\section{Laboratory 1: Introduction to MATLAB}

Students will be introduced to MATLAB concepts vital to studying EM theory. Students have had some formal programming course either in Python, $\mathrm{C}++$ or MATLAB. This will be a review of basic of MATLAB syntax and an introduction to basic signal processing concepts to be applied to EM applications such as Radar. The student will be able to take vector data and run signal processing algorithms to be used to represent targets.

\section{Laboratory 2: EM Tools and Transmission Lines}

This laboratory is an adaptation to an existing lab that has yielded successful response from students in the past [1]. Students are introduced to the RF Engineer tool bench which includes a network analyzer, oscilloscope, multimeter, and various handheld tools. Several questions guide the student to understand how to characterize a transmission line, the purpose of a transmission line, and the importance of EM tools in analysis of transmission line. Students explore the effects of phenomenon that occur at RF frequencies (such as long leads on a resistor acting as an inductor).

\section{Laboratory 3: RF Components and Theory}

Students will learn to identify block level components of a radar system that relates to both concepts learned in other EE courses and new concepts in EM. The purpose of transmission, lines, reflection coefficients, and matching networks will be analyzed and assessed to develop a high-quality radar system. This is a follow-up 
to using EM tools laboratory experiment. Each group will be responsible for characterizing transmission, reflection, VSWR of one mini-circuit component. Students will be asked questions that get them thinking about the application of electromagnetic theory to electrical engineering.

Laboratory 4: Anechoic Chamber (Part 1) and Antenna Radiation (Part 2)

This laboratory is an adaptation to an existing lab that has yielded successful response from students in the past. This is a two-week lab where students are shown the basics of the anechoic chamber and how an antenna can be measured. The student will have an understanding of why the anechoic chamber is important and be able to go through the steps to use the anechoic chamber. During the second part of the lab, students will design, simulate, and build antennas. They will measure and characterize the properties of the antennas. The student will make experimental measurements with theoretical calculations, confirm the results by measuring the operating frequency, and explain discrepancies if any. To introduce antenna and radiation concepts, the Vivaldi antenna will be used which is responsive to design changes.

Laboratory 5: Plane Wave Propagation and Polarization

Plane wave propagation and polarization is introduced along with the importance of polarization of electromagnetic radiation and how the concept can be implemented in a radar system. The student will learn how the environment plays a role in defining the polarization of a wave to obtain satisfactory results. The student will also analyze various applications of polarization such as the antenna on a car versus an antenna on a ship.

Laboratory 6: Reflection and Transmission of EM waves

Students will learn how reflection and transmission are important concepts to a radar and other EM applications. The students will conduct experiments to understand transmission and reflection of radar waves through varying medium. The students will learn how reflection and transmission are used as tools in a radar application.

Laboratory 7: Waveguides

Students will learn the theory behind designing a simple waveguide. After going through the theory, the students will learn how the waveguide can be used as a tool and when it is necessary to use a waveguide design. 
Final Lab Project: Building a radar (4 parts: Integration, Component Selection, Measurements, Analysis/Interpretation)

This lab will formally introduce the final project of building a radar to the students. The integration phase helps the student make the connection of how all the labs work together so they can build a radar system. The component selection will allow the student to design the system based on the requirements of the project. The student will be required to assemble their system and take measurements to prove their system works. Finally, the student will be required to analyze and interpret the results from the experiment.

\section{Future Work}

To further improve the overall success of the process, several topics will be addressed in future work. This includes an integration with other courses, the development of low-cost solutions for the students to work on, and development of new material each year.

The laboratory experiment has components that can be applied to various other courses within the electrical engineering curriculum. Circuit design teaches the fundamentals of waveform design, filtering, and ADC design which can be used as portions of the radar. Signal processing is vital to various algorithms used to improve signal to noise ratio. As an example, pulse compression and pulse coding techniques are used within radar to increase the resolution while obtaining a higher signal to noise ratio. Each of the EM related components of the radar can be further developed in elective courses such as antenna engineering. As an example, students can analyze and test different types of antennas such as a patch or a Vivaldi antenna to replace the $2.45 \mathrm{GHz}$ monopole.

Integrating other courses into EM course projects will foster new and improved designs based on on-going student feedback. The goal is to re-evaluate the different designs and to have students contribute to the improvement of the course as an open-source project. This will allow for students within the university to foster a pipe-line of continuous improvement so that with each generation, students are learning and getting a better experience.

One issue that professors encounter within the university is the use of information circulating among the students from previous years. This can degrade the learning and understanding of the individual student. As a counter-measure, we plan to make several changes throughout the years. The most obvious alteration is 
changing the parameters such as the frequency of operation for the radar. This seems simple, but the effect of this change will require the student to ensure they understand the frequency dependence of equations and how they affect size, weight, and power. Another alteration is to change the type of radar. We hope to develop various radar types to include for the final project such as SAR imaging radar, ground penetrating radar, and autonomous driving radar.

\section{Conclusion}

We have presented the next step in the evolution of revitalizing the electromagnetic curriculum within our university. We hope this becomes a model for other universities to use and build upon. Overall, we look to improve the understanding of students to continue to create the next wave of electrical engineers. With the integration of a hands-on approach, students will have a rich experience which will enable the understanding of a difficult topic (attainable by all students that put forth the effort to learn, that is). Ultimately, we hope to increase the number of students taking electromagnetic electives within the university which will have a direct impact on improving the quality of engineers produced for the workforce. 


\section{References:}

[1] Different Strategies for Preparing Students to Tackle the RF Engineering Challenges of Tomorrow: A Panel Discussion. (2018). In: ASEE Annual Conference and Exposition.

[2] L. E. Donohoe, J. Urbina, T. Kane, and S. G. Bilen, "ASEE 123rd Annual Conference," in Developments in the Teaching of Engineering Electromagnetics for Improvement in Student Interest and Understanding.

[3] Wankat, P. and Oreovicz, F. (1993). Teaching Engineering. [ebook] Purdue University Press, pp.168-188. Available at: https://engineering.purdue.edu/ChE/aboutus/publications/teaching_eng/chapter9.pf f.

[4] Patangia, H. (2002). A Novel Course To Provide Electrical Engineering Experience to Freshmen Students. In: American Society for Engineering Education Annual Conference \& Exposition.

[5] G. Charvat, J. Williams, A. Fenn, S. Kogon, and J. Herd. RES.LL-003 Build a Small Radar System Capable of Sensing Range, Doppler, and Synthetic Aperture Radar Imaging. January IAP 2011. Massachusetts Institute of Technology: MIT Open Course Ware, https://ocw.mit.edu. License: Creative Commons BY-NC-SA.

[6] J. R. Whinnery, "The Teaching of Electromagnetics," IEEE Transactions on Education, vol. 33, no. 1, Feb. 1990.

[7] GNU Radio. (2019). GNU Radio - The Free \& Open Source Radio Ecosystem • GNU Radio. [online] Available at: https://www.gnuradio.org/.

[8] GitHub. (2019). rseal/GnuRadar. [online] Available at: https://github.com/rseal/GnuRadar. 